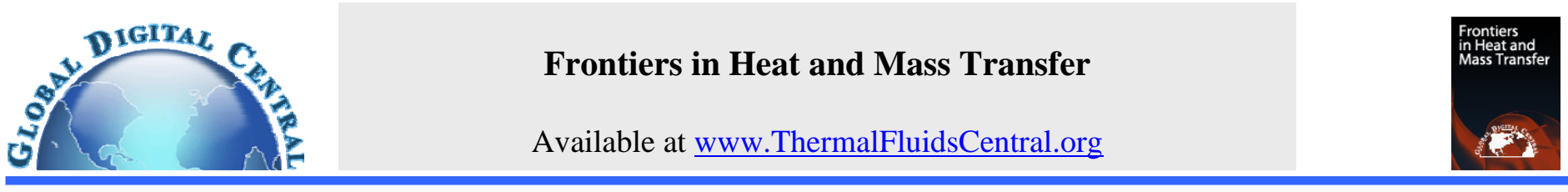

\title{
NUMERICAL SIMULATION OF NATURAL CONVECTION FROM A PAIR OF HOT CYLINDERS IN A COLD SQUARE ENCLOSURE IN DIFFERENT BOUNDARY CONDITIONS
}

\author{
Niki Rezazadeh, Rezvan Abdi* \\ Faculty of Engineering, Hakim Sabzevari University, Sabzevar, Iran
}

\begin{abstract}
This study investigates the heat transfer in the mode of natural convection from a pair of hot cylinders to a cold square enclosure. Effects of boundary conditions of the enclosure on the rate of heat transfer from a pair of isothermal hot cylinders are investigated at a Rayleigh number of $10^{5}$. The cylinders are arranged in a horizontal array at the middle height of enclosure. The commercial software, Fluent (V.6.3.26), is utilized to solve the problem using the Finite Volume Method. The streamlines as well as isothermal lines of the problem are reported. Moreover, the local Nusselt number on the walls of enclosure is calculated. The results show that the boundary conditions of the enclosure have crucial effects on the heat transfer of a pair of hot cylinders.
\end{abstract}

Keywords: heat transfer, heat flux, natural convection, circular cylinder, Nusselt number

\section{INTRODUCTION}

Heat transfer from a hot cylinder in a closed enclosure has always been of interest for researchers. In this case, the dominant mode of heat transfer is natural convection. In recent years, by developing numerical techniques and improving computer simulation programs, many researchers have been focusing on this topic to analyze the natural convection problems which were impossible to solve numerically. One of the real applications of this problem is the use of natural convection to cool the electronic circuits. Moreover, the natural convection in an enclosure has practical relevance in solar collectors, heat exchangers, refrigerator condensers, crude oil storage tanks, energy storage devices, modern crop dryers and spent fuel storage of nuclear power plants. For instance, in the case of modern crop dryers, crops are placed over the top plate covering a trench. The steam through a pipe kept inside the trench heats the air inside the trench. Also, in the case of transportation of spent nuclear fuel assembly, the assembly is put in a cask and filled with a fill gas under pressure for removing the decay heat in the fuel assembly by natural convection. The boundary conditions of the outer casting have a significant effect on the efficiency of the cooling of the fuel assembly. Similarly, the cooling effectiveness of electronic modules encapsulated inside a cabinet is affected by the wall conditions. In all the practical examples mentioned above, the thermal boundary condition has a significant effect on the mechanism of heat removal. Interestingly, the natural convection phenomena associated with the case can be studied by modelling a heated cylinder placed inside an enclosure (Roychowdhury et al, 2002).

Different studies investigated this problem can be classified into three groups: The first group has investigated the effects of geometric shape of an enclosure or the cylinder. The enclosure can be in the shape of the square (Roslan et al, 2012), triangle (Xu et al, 2012), trapeze (Da Silva et al, 2012), circle or semi-annulus (Soleimani et al, 2012). The second group has investigated the effects of numerical methods for solving the equations of natural convection in a closed enclosure. The Finite Element Method (FEM) was used by Oztop et al (2012), Immersed Boundary Method (IBM) was used by Mark et al (2013), and Finite Volume Method (FVM) was used by Mahmoodi and Sebdani (2012) to solve the governing equations of natural convection in a closed enclosure. Effects of position and size of the cylinder as well as boundary conditions were studied by the third group. Kim et al (2008) investigated the effects of Rayleigh number and vertical position of a hot circular cylinder in a cold square enclosure on the rate of heat transfer. Lee et al (2010) investigated the effects of horizontal position and diameter of a circular cylinder on the rate of heat transfer. Hussain and Hussain (2010) studied the circular cylinder in a square enclosure using limited volume method; they showed that when a constant thermal flux was applied on the cylinder surface, by moving the cylinder towards the bottom wall of the enclosure, the rate of heat transfer was decreased in low Rayleigh numbers, and, inversely, it was increased in high Rayleigh numbers. However, by moving the cylinder towards the top wall, the rate of heat transfer increased in both Rayleigh numbers.

$\mathrm{Xu}$ et al (2009) studied the natural convection of a triangular cylinder by Finite Element Method. In this study, the effects of Rayleigh number and angular position of triangular cylinder on the heat transfer were investigated. They showed that by increasing the Rayleigh number, the rate of heat transfer was increased. Moreover, when one vertex of the triangle was on the top, by increasing the angle of triangle fundus in comparison with the horizon, the rate of heat transfer of the enclosure was increased.

De and Dalal (2006) studied a square cylinder in a square enclosure. They investigated two different boundary conditions for the cylinder: constant flux and constant temperature. It was shown that in the case of constant temperature, the rate of heat transfer in the enclosure was considerably higher than the cylinder with constant flux. Moreover, they investigated the effects of changing the vertical position of the cylinder and showed that, by moving of the cylinder towards the

\footnotetext{
*Corresponding author. Email: Rezvan_Abdi@,Yahoo.com
} 
upper or lower walls of the enclosure, the rate of heat transfer would be increased in both states. It is interesting to know that when the cylinder approached the lower wall, the growing rate of heat transfer was even higher than when the cylinder was moving towards the higher wall.

Roychowdhury et al (2002) studied the effects of the ratio of circular cylinder diameter on the characterized length of the enclosure, $(D / L)$. They also investigated the effects of different boundary conditions on the rate of heat transfer. They concluded that if the two lateral walls of the enclosure having the fixed temperature and the two lower and higher walls are isolated, by increasing the ratio of $2 R / H$, the rate of heat transfer from the enclosure would increase.

Yoon et al (2010) performed a three-dimensional simulation for studying the natural convection of a sphere in a square enclosure with IBM. They reported the effects of Rayleigh number and the vertical position of the sphere on the rate of heat transfer.

The study of natural convection in a cold square enclosure with a pair of hot horizontal cylinders was studied by Park et al (2013). They investigated the effects of vertical position of cylinders by IBM for Rayleigh numbers in the range $10^{3} \leqslant R a \leqslant 10^{5}$. Moreover, the dependence of the Nusselt number on Ra was also studied. Corcione (2007) studied the natural convection from a pair of isothermal cylinders which were horizontally and vertically arranged for the Rayleigh numbers in the range $10^{2} \leqslant R a \leqslant 10^{4}$. He investigated the effects of distance between the cylinders. Corcione reported that the rate of heat transfer from a pair of isothermal cylinders may be larger or smaller than a single cylinder depending on the Ra number, the cylinder position in an array, and the geometrical arrangement of the array. Reymond et al (2008) investigated a pair of vertically aligned horizontal cylinders in three different Ra numbers $\left(2 \times 10^{6}, 4 \times 10^{6}\right.$ and $6 \times 10^{6}$ ) and three different spacing of the cylinders (corresponding to 1.5, 2 and 3 times the cylinders diameter).

Although numerous studies have been carried out on the heat transfer in the form of natural convection, the majority of them have investigated a single cylinder. Moreover, the effects of boundary conditions have not been investigated enough. Therefore, in the present study, the effects of three types of different boundary conditions on the rate of heat transfer are studied on a pair of cylinders. The commercial software, Fluent (V.6.3.26), is utilized to solve the problem using the Finite Volume Method.

The paper is organized as follows. The problem statement is given in section 2. The numerical methodology used to solve the governing equations and the validation of the numerical simulation are presented in section 3. The detailed results and the discussion are presented in section 4 and final conclusions are given in section 5 .

\section{PROBLEM STATEMENT}

As can be seen in figure 1, the problem consists of a cold square enclosure with a characterized length of $\mathrm{H}$ and a pair of hot circular cylinders with the diameter of $\mathrm{D}$. This study aims to investigate the effects of a steady natural convection on the rate of heat transfer; As shown by Park et al (2013), in a configuration similar to the one considered in the current study, the steady state condition for flow and thermal fields were observed when $10^{3} \leqslant \mathrm{Ra} \leqslant 10^{5}$. In this case, both flow and thermal fields were symmetric with respect to the vertical centerline between the two cylinders. However, when $\mathrm{Ra}=10^{6}$, the flow and thermal fields in the square enclosure became transient. Moreover, they showed that when $10^{3} \leqslant \mathrm{Ra} \leqslant 10^{4}$, heat transfer in the enclosure was governed mainly by the conduction mode. However, when $\mathrm{Ra}=10^{5}$, the role of convection in heat transfer became more significant. Therefore, in the current study, only $\mathrm{Ra}=10^{5}$ is considered.

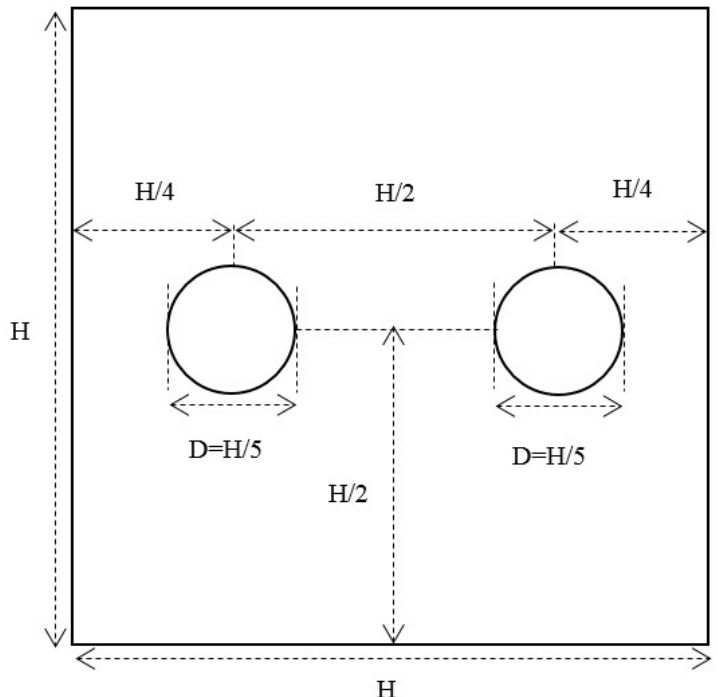

Fig. 1 Schematic diagram and computational domain of a pair of cylinders in a square enclosure.

Figure 2 shows the three types of boundary conditions which were used in this problem. Due to the symmetrical configuration, only half of the figure was considered. The temperature of cylinder surface was equal to $T_{h}$ and remained constant, and the square enclosure was located in lower temperature and under three different boundary conditions. The first boundary condition, case 1, was isothermal condition on the right wall of the enclosure, and the other walls had adiabatic condition. The second boundary condition, case 2, was the isothermal condition for all the enclosure walls. The third boundary condition, case 3, was the isothermal condition on the top wall of the enclosure and the other walls had adiabatic condition.

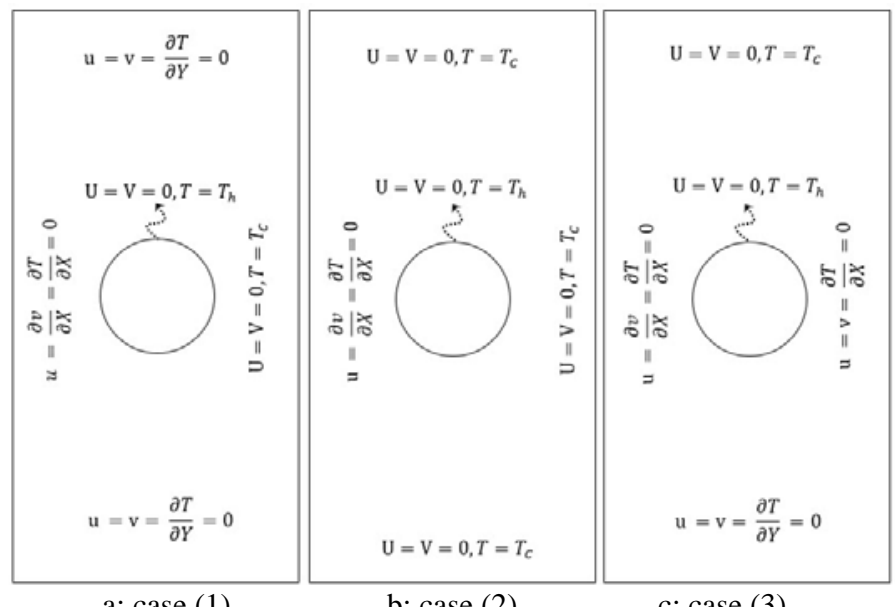

Fig. 2 The boundary conditions.

In this simulation, air was used as the fluid domain and it was assumed that the properties of air were constant, except density. A varying density was considered by the incompressible Boussinesq approximation in the buoyancy term of Navier-Stokes equation. In this two-dimensional analysis, the gravitational acceleration acted in the negative y-direction, the flow was laminar and steady, and the radiation effects were neglected. The meshed domain is shown in figure 3 where a coarse grid is shown for clarity. 


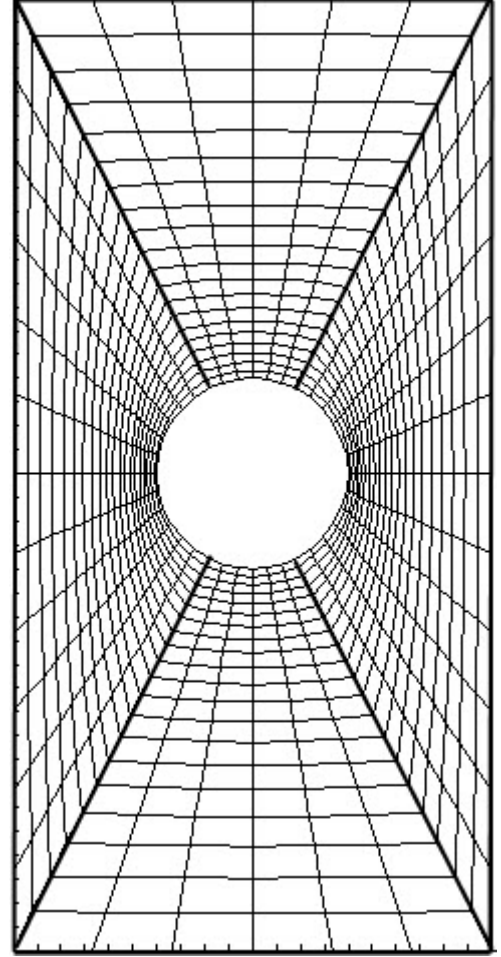

Fig. 3 Meshed domain. A course grid is shown for clarity.

\section{THE NUMERICAL METHODOLOGY AND VALIDATION}

\subsection{Governing Equations}

The following dimensionless variables are defined to state the governing equations in the dimensionless form:

$$
X=x / H, Y=y / H, U=u H / \alpha, V=v H / \alpha, P=\left(p H^{2}\right) /\left(\rho \alpha^{2}\right) \text {, }
$$$$
\theta=\left(T-T_{c}\right) /\left(T_{h}-T_{c}\right)
$$

where $u$ and $v$ are the velocity components in the $x$ and $y$ directions, respectively, $\rho$ is the density, $\mu$ is the dynamic viscosity, $p$ is the static pressure, $\mathrm{H}$ is the height of enclosure, $\alpha$ is the thermal diffusivity, $T$ is the temperature, $\mathrm{T}_{\mathrm{h}}$ is the hot temperature at the cylinder surface and $T_{c}$ is the cold temperature at the enclosure walls. It should be noted that $T_{h}$ must be obtained according to:

$$
T_{h}=(\text { Ra. } \alpha . \vartheta) /\left(\text { g. } \beta . H^{3}\right)+T_{c}
$$

where $\vartheta(=\mu / \rho$ and $\mu$ is the dynamic viscosity) is the kinematic viscosity, and $R a$ ( $=g \beta\left(T_{h}-T_{c}\right) H^{3} / \alpha \vartheta$ and $\beta$ is the Volumetric coefficient of thermal expansion and $g$ is the gravitational acceleration) is the Rayleigh number which is equal to $10^{5}$ in this paper.

The governing equations are the continuity, momentum (in both $x$ and $y$ directions), and the energy which are defined in the non-dimensional form in (3)-(6) formulas, respectively.

$$
\begin{aligned}
& (\partial U / \partial X)+(\partial V / \partial Y)=0 \\
& U(\partial U / \partial X)+V(\partial U / \partial Y)=-(\partial P / \partial X)+\operatorname{Pr}\left(\left(\partial^{2} U\right) /\left(\partial X^{2}\right)+\left(\partial^{2} U\right) /\left(\partial Y^{2}\right)\right)
\end{aligned}
$$

$U(\partial V / \partial X)+V(\partial V / \partial Y)=-(\partial P / \partial Y)+\operatorname{Pr}\left(\left(\partial^{2} V\right) /\left(\partial X^{2}\right)+\left(\partial^{2} V\right) /\left(\partial Y^{2}\right)\right)+$ $\operatorname{Ra} \times \operatorname{Pr} \times \theta$

$$
U(\partial \theta / \partial X)+V(\partial \theta / \partial Y)=\left(\partial^{2} \theta\right) /\left(\partial X^{2}\right)+\left(\partial^{2} \theta\right) /\left(\partial Y^{2}\right)
$$

where $\operatorname{Pr}(=\vartheta / \alpha)$ is the Prandtl number which is equal to 0.71 in this paper corresponding to that of air.

The dimensionless parameter of heat transfer is Nusselt number, $(\mathrm{Nu})$, which has been computed according to:

$$
N u=(\partial \theta) /\left.\partial n\right|_{\text {wall }}
$$

where $n$ is the normal direction with respect to the walls.

The mean value of Nusselt number is defined according to:

$$
\overline{N u}=\frac{1}{a} \int_{0}^{a} N u d s
$$

where $\mathrm{s}$ is the distance along the enclosure walls.

\subsection{Finite Volume Scheme}

The system of two-dimensional equations (3-6) was solved using the Fluent software. Fluent is a software for solving problems defined by partial differential equations (PDEs) using Finite Volume Method (FVM). The case was set with a pressure based, implicit and steady solver with Green-Gauss Cell Based gradient treatment. Gradients of solution variables were used for evaluating of diffusive fluxes, velocity derivatives, and for higher-order discretization schemes. When using the pressure-based solver, a numerical algorithm is required to derive an equation for pressure (or pressure correction). This algorithm which is called Pressure-velocity coupling, uses a combination of continuity and momentum equations. In this study, the SIMPLEC (SIMPLEConsistent) algorithm was used to relate the velocity field to the pressure field with relaxation factors of 0.3 for pressure, 0.1 for velocity and 1 for temperature. The discretization of the equations is implemented using a Power-law differencing scheme in a staggered grid system. Velocities are stored at cell surfaces and scalars, such as pressure and volume fraction, are stored at the center of the cell. In order to discretize the pressure terms, PRESTO (PREssure STaggering Option) scheme was used. It uses the discrete continuity balance for a "staggered" control volume about the face to compute the "staggered" (i.e., face) pressure (Patankar, 1980; FLUENT, 2013).

\subsection{Grid Independence Tests}

The grid independence tests were conducted on the natural convection of a circular cylinder in a rectangular enclosure where all walls of the enclosure had a cold constant temperature. Four different grids, namely, $60 \times 60,80 \times 80,100 \times 100$ and $120 \times 120$ are employed for the numerical calculations. A comparison of surface-averaged Nusselt number of enclosure for four different mesh resolutions is shown in Table 1. It can be seen that the $100 \times 100$ grid is sufficiently fine for the numerical calculation and was chosen for all subsequent simulations.

Table 1 Grid independence test for natural convection of a circular cylinder in a rectangular enclosure.

\begin{tabular}{|c|c|c|c|c|}
\hline Grid & $30 \times 60$ & $40 \times 80$ & $50 \times 100$ & $60 \times 120$ \\
\hline$\overline{\mathrm{W}}$ & 1.80 & 1.75 & 1.754 & 1.754 \\
\hline
\end{tabular}

\subsection{Validation of the Finite Volume Model}

The numerical model is validated by simulating the natural convection of a circular cylinder in an enclosure, where the cylinder surface had a constant high temperature and the walls of enclosure were in the constant low temperature. As shown in Table 2, the current result compares well with the numerical results of other studies, including Khozeymehnejad and Mirbozorgi (2012) and Kim et al (2008). The small differences among these results could be attributed to the different numerical approaches which have been implemented in each study. 
Table 2 Comparison of surface-averaged Nusselt number of square enclosure with the benchmark result.

\begin{tabular}{|c|c|c|c|c|}
\hline \multirow{2}{*}{$\mathrm{Ra}$} & $\begin{array}{c}\text { Khozeymehnejad } \\
\text { \& Mirbozorgi } \\
\text { (FVM) }\end{array}$ & $\begin{array}{c}\text { Kim et al } \\
\text { (IBM) }\end{array}$ & $\begin{array}{c}\text { Our } \\
\text { simulation } \\
\text { (FVM) }\end{array}$ & $\begin{array}{c}\text { Deviation } \\
\text { (\%) }\end{array}$ \\
\hline $10^{5}$ & 2.58 & 2.51 & 2.486 & $0.95-3.6$ \\
\hline
\end{tabular}

\section{RESULTS AND DISCUSSIONS VALIDATION}

\subsection{Effects of Boundary Conditions on Isothermal Lines}

Figures 4 shows the effects of boundary conditions on isothermal lines of the natural convection of a pair of cylinders in different boundary conditions. As can be seen in figure 4, the density of the isothermal lines on the top of the cylinder surface is lower than other sides of the cylinder indicating that the natural convection was the dominant mechanism of heat transfer in this problem. The upward flow which was generated on either side of the cylinder resulted in decreasing the density of isothermal lines on the top of cylinder surfaces.

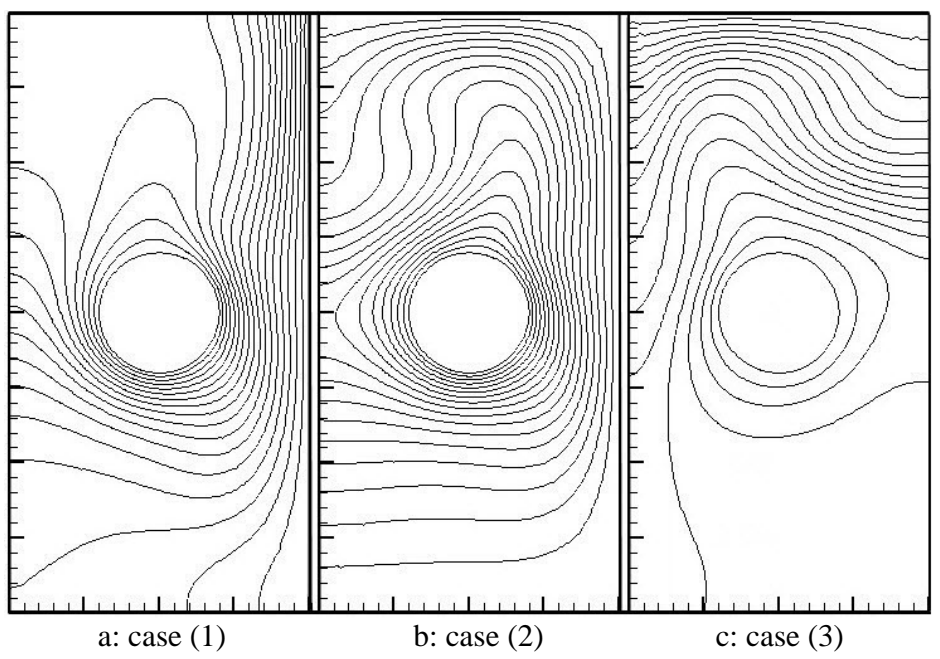

Fig. 4 Isothermal lines for a pair of hot cylinders in different boundary conditions.

\subsection{Effects of Boundary Conditions on Streamlines}

Figure 5 shows the streamlines near the hot cylinder in the enclosure in different boundary conditions. As stated before, the cylinder was warmer than the enclosure, hence, an upward flow was generated on both right and left sides of the cylinder. It can be seen that in all three cases, in addition to the main circulating flow, three vortices were formed. The main circulating flow and the three vortices in case 1 and case 2 had the same rotational direction; however; in case 3 an opposite corresponding directions was observed. It can be seen from figure 5(a), in case 1 a clockwise circulating main flow was generated. The generating mechanism of this main circulating flow is that when the upward flow (at the left side of the cylinder) contacted the upper wall of the enclosure, it moved towards the lateral cold wall (on a horizontal path to the right of the enclosure). Then, the flow contacted the lateral cold wall and moved downward (on a vertical path). This downward flow had leaded to a change in the direction of the upward flow to an oblique path. Hence, a vacant space was generated on the right top of the enclosure and consequently a vortex was generated in this vacant space. The reason for this phenomena is that some of the upward streamlines near the left side of the enclosure, after contacting the upper enclosure wall, deviated to the left side of the enclosure (due to the lower pressure of this region). Hence, a counter clockwise vortex was generated in this vacant space. Moreover, the downward flow (near the right wall of the enclosure) affected the upward flow which was generated on the right side of the cylinder and led to its return. The reverse flow caused generation of two clockwise vortices on the right side of the cylinder. When the three walls were on the constant cold temperature (figure 5(b)), a stronger downward flow was generated. Because of this stronger downward flow, a bigger vortex was generated on the left top of the enclosure. When only the upper wall was on the constant cold temperature, figure 5(c), the flow rotational direction in the enclosure was changed from clockwise (at case 1 and case 2) to counter clockwise (at case 3). Hence, a downward flow was generated on the left side of the enclosure. Therefore, the upper vortex was generated on the right top side of the enclosure and the two smaller vortices were generated at the left side of the cylinder. All these vortices had an opposite direction to the corresponding vortices of case 1 and case 2 .

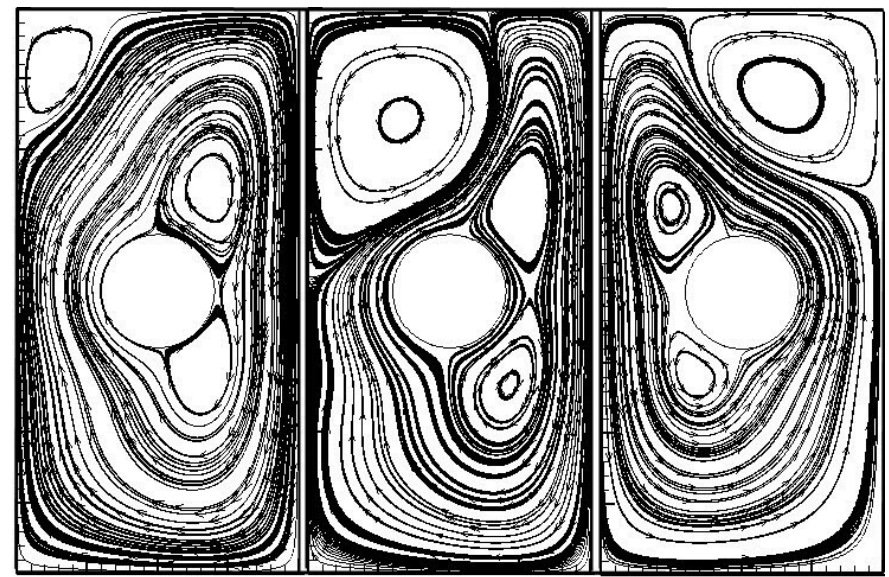

a: case (1)

b: case (2)

c: case (3)

Fig. 5 Streamlines for a pair of hot cylinders in different boundary conditions.

\subsection{Effects of Boundary Conditions on Local Nusselt Number on the Walls of the Enclosure}

Figure 6 shows the effects of the boundary conditions on the local Nusselt number $(\mathrm{Nu})$ on the walls of the enclosure along the dimensionless length $(S / H)$ where $\mathrm{S}$ is the distance from the left top wall of the enclosure as shown in figure 6. It can be seen that by moving along the $\mathrm{S}$ (from $A$ to $B$ ), the $\mathrm{Nu}$ number of case (2) is increased and reached the maximum value at the middle of the top wall (point $B$ ). After that, it decreases until it reaches approximately zero value at point $C$. As can be seen in figure 4(b), the isothermal lines are compressed at point $B$, hence, the maximum of Nu number is achieved at this point. After that, the Nu number reaches to the zero value at the point $C$ due to the sharp corner of the enclosure where the streamlines can't flow on it (figure 5(b)). Along the left wall of the enclosure (from $C$ to $E$ ), $\mathrm{Nu}$ initially increases until it reaches to a relative maximum value at point $D$, then it decreases. Due to the existence of the cylinder, the stream space decreases near point $D$ and causes that the isothermal lines become compressed at this point. Thus, the Nu number reaches the relative maximum value at point $D$. Along the bottom wall of the enclosure (from $E$ to $G$ ), the Nu number increases. However, it has a lower value compared to the Nu number of the top wall. As stated before, the natural convection was the dominant mode of heat transfer in the enclosure, hence, it amplifies the flow and heat transfer on the top half of the enclosure compared to the lower half.

The Nu number along the top wall for case 3 has a similar trend compared to case 2 at corresponding dimensionless length. However, case 3 has a higher value of $\mathrm{Nu}$ number and reaches the maximum 
value at a shorter distance. Due to the adiabatic condition of the right and bottom walls, the Nu number is equal to zero on these walls.

As can be seen in figure 4(a), the isothermal lines are compressed near point $C$. Hence, the Nu number on the right wall of the enclosure for case 1 has a high value at this point (figure 6). After that, the density of isothermal lines is decreased (from $C$ to $E$ ). Hence, Nu number is decreased along this path.

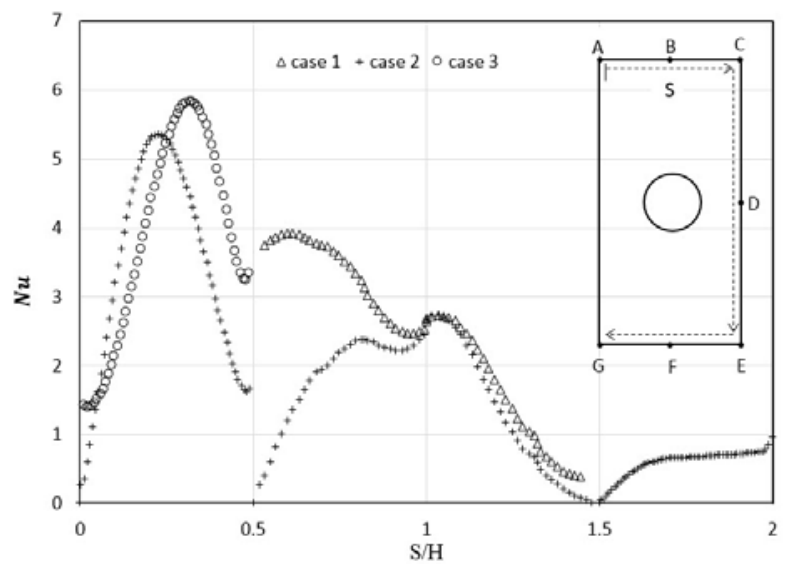

Fig. 6 Local Nu distribution on the walls of the enclosure.

Table 3 shows the surface-averaged Nusselt number of each wall and the sum of them for each case. The surface-averaged Nusselt number of the top wall $\left(\overline{N u}_{A-c}\right)$ of case 3 is greater than the corresponding value of case 2. Moreover, the surface-averaged Nusselt number of the right wall $\left({\overline{\sqrt{w_{G}}}}_{\mathrm{E}-\mathrm{E}}\right)$ of case 1 is greater than the corresponding value of case 2 . Due to the adiabatic boundary condition of the two walls in case 1 and case 3 , the heat transfer occurs only on the other walls i.e., the top wall of case 3 and the right wall of case 1 . Hence, the surface-averaged Nu number on these walls in case 3 and case 1 is greater than the corresponding wall of case 2, respectively, where heat transfer occurs on all walls of the enclosure. Moreover, this occurrence leads to an increase in the surface-averaged Nusselt number of square enclosure ( $\bar{W}$, i.e., the sum of surface-averaged Nusselt number of all walls of the enclosure) in case 2, compared to the other two cases.

Table 3 Surface-averaged Nusselt number of square enclosure, $\sqrt{\mathrm{nu}}$.

\begin{tabular}{|c|c|c|c|}
\hline $\begin{array}{c}\text { Averaged } N u \\
\text { number }\end{array}$ & Case1 & Case2 & Case3 \\
\hline$\overline{\mathrm{N}}_{-\boldsymbol{-}}$ & 0 & 0.843 & 0.947 \\
\hline$\overline{\mathrm{Nu}}_{-\varepsilon}$ & 1.1317 & 0.761 & 0 \\
\hline$\overline{\mathrm{Nu}}_{\boldsymbol{E}-\boldsymbol{\varepsilon}}$ & 0 & 0.15 & 0 \\
\hline$\overline{\mathrm{Nu}}$ & 1.1317 & 1.754 & 0.947 \\
\hline
\end{tabular}

\section{SUMMARY AND CONCLUSIONS}

A numerical investigation of the heat transfer in the mode of natural convection from a pair of circular cylinders in a square enclosure has been performed in this work. The cylinders were arranged in a horizontal array at the middle height of enclosure. The effects of boundary conditions on the local Nusselt number of the enclosure walls were investigated in the Rayleigh of $10^{5}$. It has been shown that the rate of heat transfer of a pair of cylinders in an enclosure can be effectively influenced by means of the boundary conditions of the enclosure. The heat transfer of a wall can be increased when the other two walls were in the adiabatic boundary condition (case1 and case 3), however, in these cases, the heat transfer of the enclosure was lower than case 1 , where all walls had a constant cold temperature. The current investigation sheds light on the future developments of efficient heat transfer controlling methods for two or even more cylinders in an enclosure.

\section{NOMENCLATURE}

$\begin{array}{ll}g & \text { gravitational acceleration }\left(\mathrm{m} / \mathrm{s}^{2}\right) \\ H & \text { height of the enclosure }(\mathrm{m}) \\ L & \text { characterized length of the enclosure } \\ R & \text { Radius of circular cylinder }(\mathrm{m}) \\ T & \text { temperature }(\mathrm{K}) \\ P & \text { static pressure }(\mathrm{pa}) \\ S & \text { distance along the square enclosure, }(\mathrm{m}) \\ N u & \text { Nusselt number } \\ R a & \text { Rayleigh number } \\ \mathrm{Pr} & \text { Prandtl number } \\ D & \text { cylinder diameter }(\mathrm{m}) \\ u & \text { velocity components in } x \text {-direction }(\mathrm{m} / \mathrm{s}) \\ v & \text { velocity components in } y \text {-direction }(\mathrm{m} / \mathrm{s}) \\ x & \text { Cartesian coordinates in horizontal direction }(\mathrm{m}) \\ y & \text { Cartesian coordinates in vertical direction }(\mathrm{m}) \\ n & \text { normal direction with respect to the walls } \\ & \text { Average Nusselt number } \\ \text { Greek Symbols } & \text { volumetric expansion coefficient }\left(\mathrm{K}^{-1}\right) \\ \beta & \text { dynamic viscosity }(\mathrm{kg} / \mathrm{m} . \mathrm{s}) \\ \mu & \text { kinematic viscosity }\left(\mathrm{m}^{2} / \mathrm{s}\right) \\ \vartheta & \text { density }\left(\mathrm{kg} / \mathrm{m}^{3}\right) \\ \rho & \text { dimensionless temperature } \\ \theta & \text { thermal diffusivity }\left(\mathrm{m}^{2} / \mathrm{s}\right) \\ \alpha & \text { hot wall } \\ \text { Superscripts } & \text { cold wall } \\ h & \text { free-stream } \\ c & \text { initial condition } \\ \infty & \end{array}$

\section{REFERENCES}

Corcione, M., 2007, "Interactive Free Convection from a Pair of Vertical Tube-Arrays at Moderate Rayleigh Numbers,” International Journal of Heat and Mass Transfer, 50(5), 1061-1074.

http://dx.doi.org/10.1016/j.ijheatmasstransfer.2006.07.034

Da Silva, A., Fontana, É., Mariani, V. C., \& Marcondes, F., 2012, "Numerical Investigation of Several Physical and Geometric Parameters in the Natural Convection into Trapezoidal Cavities," International Journal of Heat and Mass Transfer, 55(23), 6808-6818. http://dx.doi.org/10.1016/j.ijheatmasstransfer.2012.06.088

De, A. K., \& Dalal, A., 2006, "A Numerical Study of Natural Convection around a Square, Horizontal, Heated Cylinder Placed in an Enclosure,” International Journal of Heat and Mass Transfer, 49(23), 4608-4623.

http://dx.doi.org/10.1016/j.ijheatmasstransfer.2006.04.020

FLUENT v. 6.3.26, User’s Guide 2013.

Hussain, S. H., \& Hussein, A. K., 2010, "Numerical Investigation of Natural Convection Phenomena in a Uniformly Heated Circular 
Cylinder Immersed in Square Enclosure Filled with Air at Different Vertical Locations," International Communications in Heat and Mass Transfer, 37(8), 1115-1126.

http://dx.doi.org/10.1016/j.icheatmasstransfer.2010.05.016

Khozeymehnezhad, H., \& Mirbozorgi, S. A., 2012, “Comparison of Natural Convection around a Circular Cylinder with a Square Cylinder Inside a Square Enclosure," Journal of Mechanical Engineering and Automation, 2(6), 176-183.

http://dx.doi.org/10.5923/j.jmea.20120206.08

Kim, B. S., Lee, D. S., Ha, M. Y., \& Yoon, H. S., 2008, “A Numerical Study of Natural Convection in a Square Enclosure with a Circular Cylinder at Different Vertical Locations," International Journal of Heat and Mass Transfer, 51(7), 1888-1906.

http://dx.doi.org/10.1016/j.ijheatmasstransfer.2007.06.033

Lee, J. M., Ha, M. Y., \& Yoon, H. S., 2010, "Natural Convection in a Square Enclosure with a Circular Cylinder at Different Horizontal and Diagonal Locations," International Journal of Heat and Mass Transfer, 53(25), 5905-5919.

http://dx.doi.org/10.1016/j.ijheatmasstransfer.2010.07.043

Mark, A., Svenning, E., \& Edelvik, F., 2013, “An Immersed Boundary Method for Simulation of Flow with Heat Transfer," International Journal of Heat and Mass Transfer, 56(1), 424-435.

http://dx.doi.org/10.1016/j.ijheatmasstransfer.2012.09.010

Mahmoodi, M., \& Sebdani, S. M., 2012, "Natural Convection in a Square Cavity Containing a Nanofluid and an Adiabatic Square Block at the Center," Superlattices and Microstructures, 52(2), 261-275.

http://dx.doi.org/10.1016/j.spmi.2012.05.007

Öztop, H. F., Rahman, M. M., Ahsan, A., Hasanuzzaman, M., Saidur, R., Al-Salem, K., \& Rahim, N. A., 2012, "MHD Natural Convection in an Enclosure from Two Semi-Circular Heaters on the Bottom Wall," International Journal of Heat and Mass Transfer, 55(7), 1844-1854. http://dx.doi.org/10.1016/j.ijheatmasstransfer.2011.11.037

Patankar, S., 1980, Numerical Heat Transfer and Fluid Flow, CRC Press.

Park, Y. G., Ha, M. Y., \& Yoon, H. S., 2013, "Study on Natural Convection in a Cold Square Enclosure with a Pair of Hot Horizontal Cylinders Positioned at Different Vertical Locations,” International Journal of Heat and Mass Transfer, 65, 696-712.

\section{http://dx.doi.org/10.1016/j.ijheatmasstransfer.2013.06.059}

Reymond, O., Murray, D. B., \& O’Donovan, T. S., 2008, "Natural Convection Heat Transfer from Two Horizontal Cylinders," Experimental Thermal and Fluid Science, 32(8), 1702-1709. http://dx.doi.org/10.1016/j.expthermflusci.2008.06.005

Roslan, R., Saleh, H., \& Hashim, I., 2012, "Effect of Rotating Cylinder on Heat Transfer in a Square Enclosure Filled with Nanofluids," International Journal of Heat and Mass Transfer, 55(23), 7247-7256. http://dx.doi.org/10.1016/j.ijheatmasstransfer.2012.07.051

Roychowdhury, D. G., Das, S. K., \& Sundararajan, T., 2002, "Numerical Simulation of Natural Convective Heat Transfer and Fluid Flow Around a Heated Cylinder Inside an Enclosure," Heat and Mass Transfer, 38(7-8), 565-576. http://dx.doi.org/doi:10.1007/s002310100210

Soleimani, S., Sheikholeslami, M., Ganji, D. D., \& Gorji-Bandpay, M., 2012, "Natural Convection Heat Transfer in a Nanofluid Filled SemiAnnulus Enclosure," International Communications in Heat and Mass Transfer, 39(4), 565-574. http://dx.doi.org/10.1016/j.icheatmasstransfer.2012.01.016

Xu, X., Yu, Z. T., Hu, Y. C., Fan, L. W., \& Cen, K. F., 2012, "Transient Natural Convective Heat Transfer of a Low-Prandtl-Number Fluid from a Heated Horizontal Circular Cylinder to its Coaxial Triangular Enclosure," International Journal of Heat and Mass Transfer, 55(4), 995-1003. http://dx.doi.org/10.1016/j.ijheatmasstransfer.2011.10.011

Xu, X., Sun, G., Yu, Z., Hu, Y., Fan, L., \& Cen, K., 2009, “Numerical Investigation of Laminar Natural Convective Heat Transfer from a Horizontal Triangular Cylinder to its Concentric Cylindrical Enclosure," International Journal of Heat and Mass Transfer, 52(13), 3176-3186.

http://dx.doi.org/10.1016/j.ijheatmasstransfer.2009.01.026

Yoon, H. S., Yu, D. H., Ha, M. Y., \& Park, Y. G., 2010, “ThreeDimensional Natural Convection in an Enclosure with a Sphere at Different Vertical Locations,” International Journal of Heat and Mass Transfer, 53(15), 3143-3155.

http://dx.doi.org/10.1016/j.ijheatmasstransfer.2010.03.013 